\title{
Review of: "Dopamine Neurons Evaluate Natural Fluctuations in Performance Quality"
}

\author{
Wei Meng ${ }^{1}$ \\ 1 Jiangxi Science \& Technology Normal University
}

Potential competing interests: The author(s) declared that no potential competing interests exist.

This study used a songbird model, Zebra finches, to show for the first time that the activity of dopamine neurons in the ventral tegmental area (VTA) is associated with fluctuations in a natural behavior. Dopamine has been confirmed to play a key role in ongoing behavior ${ }^{[1]}$. However, revealing the mechanism of dopamine regulating ongoing behavior, especially natural behavior, is a challenge in both basic and clinical research. Songbirds' song behavior is regulated by a clear Song Control System, a set of interconnected nuclei, and their songs can be quantitatively analyzed by specialized acoustic software, making songbirds an excellent model for studying behavior regulation. It is proven that dopamine affects the production and performance of songbirds singing ${ }^{[2]}$. In 2016, Gadagkar et al. published an excellent study demonstrating that dopamine neurons in VTA encode performance error in singing zebra finches ${ }^{[3]}$. In their present study, Gadagkar and colleagues developed a Gaussian Process Regression analysis (to be honest, I am not familiar with the derivation and application of the method) and concluded that the spontaneous firing of $X$ area-projecting dopamine neurons in VTA is related to natural song fluctuations in a manner of evaluation. This important conclusion sheds light on the underlying regulatory role of basal ganglia-inputting dopamine in natural behavior.

In the meantime, I have a few questions about this manuscript and research finding.
1. Figure 1
$(A),(B)$ and $(C$
(C) are almost identical to Figure 1
$(A),(B)$ and
(E) in Gadagkar et al.'s paper published in Science in $2016^{[3]}$.

2. If Figure $1(D)$ and $(E)$ are the experimental results of this research, why are they indicated in "Introduction" but not in "Results" section?

3. Some figure legends lack details, such as Figure 1 (A), (B), (C) and (E). Another question is, in Figure 4 (E), which point is "the blue point" described in Line 361.

4. It has been reported that dopaminergic projections from midbrain to primary motor cortex mediate motor skill learning in rats ${ }^{[4]}$. In songbirds, in addition to $X$ area, song premotor nuclei HVC and RA, sensorimotor cortical analogues, also receive dopaminergic projections from midbrain ${ }^{[5,6]}$. A study has shown that dopamine released into HVC promotes song imitation in juvenile zebra finches ${ }^{[7]}$. At the same time, excitatory synaptic transmission received by the projection neurons in song premotor nucleus RA was confirmed to be regulated by dopamine ${ }^{[8]}$. I would like to know whether the authors plan to examine, or have examined, the relationship between the activity of HVC-projecting and RA-projecting 
dopamine neurons and song behavior, and how to consider the potential dopamine interactions of these three nuclei.

Sincerely,

Wei Meng

\section{References}

1. Berke JD. What does dopamine mean? Nat Neurosci. 2018, 21(6): 787-793.

2. Hoffmann LA, Saravanan V, Wood AN, He L, Sober SJ. Dopaminergic Contributions to Vocal Learning. J Neurosci. 2016, 36(7): 2176-2189.

3. Gadagkar V, Puzerey PA, Chen R, Baird-Daniel E, Farhang AR, Goldberg JH. Dopamine neurons encode performance error in singing birds. Science. 2016, 354(6317): 1278-1282.

4. Hosp JA, Pekanovic A, Rioult-Pedotti MS, Luft

AR. Dopaminergic projections from midbrain to primary motor cortex mediate motor skill learning. J Neurosci. 2011, 31(7): 2481-2487.

5. Appeltants D, Absil P, Balthazart J, Ball GF. Identification of the origin of catecholaminergic inputs to HVc in canaries by retrograde tract tracing combined with tyrosine hydroxylase immunocytochemistry. J Chem Neuroanat. 2000, 18(3): 117-133.

6. Appeltants D, Ball GF, Balthazart J. The origin of catecholaminergic inputs to the song control nucleus RA in canaries. Neuroreport. 2002, 13(5): 649-653.

7. Tanaka M, Sun F, Li Y, Mooney R. A mesocortical dopamine circuit enables the cultural transmission of vocal behavior. Nature. 2018, 563(7729): 117-120.

8. Wang S, Liu S, Wang Q, Sun Y, Yao L, Li D, Meng W. Dopamine Modulates Excitatory Synaptic Transmission by Activating Presynaptic D1-like Dopamine Receptors in the RA Projection Neurons of Zebra Finches. Front Cell Neurosci. 2020, 14: 126. doi: 10.3389/fncel.2020.00126. 